\title{
New I-Ching and Sports Database
}

\section{Hongwen Cheng}

Department of Medicine, The Third People's Hospital of Zhongxiang City, Zhongxiang, China

Email: chenghwn@aliyun.com

How to cite this paper: Cheng, H.W. (2021) New I-Ching and Sports Database. Open Access Library Journal, 8: e7321. https://doi.org/10.4236/oalib.1107321

Received: March 16, 2021

Accepted: May 25, 2021

Published: May 28, 2021

Copyright $\odot 2021$ by author(s) and Open Access Library Inc.

This work is licensed under the Creative Commons Attribution International License (CC BY 4.0).

http://creativecommons.org/licenses/by/4.0/

\begin{abstract}
In order to adapt to the development of modern science and technology, I-Ching needs to be modified. In order to make it easy to understand, and become a powerful "science" tool for our learning, thinking and practice, according to the characteristics of our thinking, I tried to revise the images of the hexagrams, gua ci and lines of the I-Ching. Then when we use I-Ching to thought; it will let us examine various factors, changes and relationships of various influences, so that we can solve problems faster, better and more creatively.
\end{abstract}

\section{Subject Areas}

Philosophy

\section{Keywords}

New I-Ching, Images, Main Gua, Change Gua, Movement Database

\section{1. 前言}

我们知道《易经》被誉为诸经之首 “大道之源” [1], 是中华民族五千年 智慧的结晶。但易经是从占卜发展而来的, 虽经过后世学者的不断修改发展, 已经远远脱离占卜的范畴, 但它的修改仍不够彻底, 占卜对它仍然存在强烈 的影响, 从易经对卦、爻的各种解释仍然给人牵强附会、缺乏逻辑的感觉, 就可以看出。因而人们都认为易经晦澀难懂, 给人以神秘之感也就好理解了。 同时, 易经理论发展缓慢, 与现代科技缺乏很好的衔接, 也是因为我们不敢 真正打破 “老祖宗” 的条条框框, 对它进行大的修改。我们需要对它进行大 的修改, 使它摆脱占卜的不利影响, 彻底成为一门真正的科学。

我们可以这样理解易经, 易经是古人建立的力图包罗万象的运动数据库, 虽然在建立与完善过程中存在各种问题，但对我们现在建立包罗万象的运动 
数据库仍然有很大的借鉴意义。新易经部分借鉴了易经的理念与方法来建立 包罗万象的运动数据库。

科学发展到现在, 在复杂性问题的研究上陷入困境, 重要的原因之一是 计算数学的发展陷入了瓶颈, 比如蛋白质链的折叠计算, 局部生态环境的发 展变化的计算等等。新易经建立的运动数据库及它建立的方法有助于我们对 这些复杂性问题的解决。比如在解决一领域中某一复杂性问题时, 我们一方 面可以通过新易经调取相似运动来参考, 另一方面在这个复杂性问题被解决 后, 我们所获得的运动数据又可以归入新易经中的运动数据库中, 为今后解 决其它领域的复杂性运动提供帮助。新易经它是为未来的解决复杂性问题的 计算数学准备的。

新易经包罗万象, 容易理解, 富有逻辑性, 有了它我们在作卦时会自觉 的用全面、整体的观点, 普遍联系的观点, 变化发展的观点, 来考察各种影 响因素及对象的发展变化规律及相互关系, 使我们能更好、更快、更有创造 性地解决问题。

本文，通过我们对易经及现代科技的理解，吸取易经的精华，对易经进 行了全面修改，从而使它成为与传统易经存在明显区别的 “新易经”。通过 新易经作卦就是构建运动数据, 它存在明确而简单的标准与逻辑。这样获取 的标准运动数据库, 一方面使新易经相对于易经更加通俗易懂, 另一方面通 过对运动数据库的应用, 使它成为我们学习、思考、工作实践的有力 “科学” 工具。我在这里的独立修改只是告诉大家要如何修改, 起到抛砖引玉的作用, 远不是最终的结果。它的修改完善是个庞大的系统工程, 需要大家的共同参 与完善。

\section{2. 易经的修改}

修改的目的：1）力求简洁明了, 使它通俗易懂, 富有逻辑性。一看卦象 就会对所考察的对象有一个大概的了解。2) 在作卦的过程中会促使我们用全 面、整体的观点, 普遍联系的观点, 变化发展的观点, 来考察各种影响因素 及对象的发展变化规律及相互关系。从而作出相对理想的卦象。3) 作出卦后 又会通过相应卦象的各种注、辞及经验描述而更好、更快、更有创造性的解 决问题。我们作卦的目的是为了更好的思考与解决问题, 并通过卦象能给我 们以各种启迪。

我的修改是以阴阳为基础, 对卦、爻重新进行了排列、解释, 并增加了 一些新的内容，从而使它们更容易理解与应用，也更有逻辑性。

\section{1. 新易经的结构功能及原因}

\subsection{1. 卦图的组成结构、原因}

我们考察的任何对象，之所以是考察时的状态，一般情况下都是因为在 存在使这种状态向某方向运动发展的因素的同时，必然存在使这种状态向相 反方向运动发展的因素。那些使对象存在发展、向上发展、向前发展……的 因素集合称之为阳用 “---” 来表示，叫阳爻，那些使对象不存在发展或者向 
下发展、向后发展……的因素集合称之为阴, 用 “- - ” 来表示, 叫阴爻。阳 爻与阴爻是卦象的基本元素。

为了包含所有强烈影响或可能强烈影响对象运动发展的因素及反应它们 大概的作用情况, 我将一个卦分为阳中阴三大部分, 其中中性属性集合部分 是易经所没有的, 是我新加的。

如图 1: 一个卦分三大部分, a 部分代表阳性属性集合, b 部分代表中性 属性集合, $\mathrm{c}$ 部分代表阴性属性集合。中性属性集合包含的影响因素是那些对 对象的运动发展暂时没有什么影响但随时可能发生影响的因素。如图 1: a 、

b、 $c$ 又分别包括阳、中、阴三部分, 它们又分别是一卦。如图 2: 每一卦又 由九个爻组成, 而每个爻又可以看成一卦。如图 2: 1 所对应的爻, 代表的是 这个对象向一方向发展的主要正性因素, 当 1 爻是阳爻时, 表示正性发展的 力量强, 而当 1 爻是阴爻时, 表示正性发展的力量较弱。 7 所代表的是主要 负性因素，同理，当 7 爻是阳爻时，表示负性发展的力量强，而当 7 爻是阴 爻时, 表示负性发展的力量较弱; 2 与 8 所代表的是次要因素(也就是相对中 性因素)。2 爻为阳爻, 表示次要因素向主要因素转换的倾向较强, 2 爻为阴 爻, 表示次要因素向主要因素转换的倾向较弱。8 爻为阳爻, 表示次要负性 因素向主要负性因素转换的倾向较强, 8 爻为阴爻, 表示次要因素向主要因 素转换的倾向较弱; 3,9 分别代表促进所在卦向中性或者相反方向发展的因

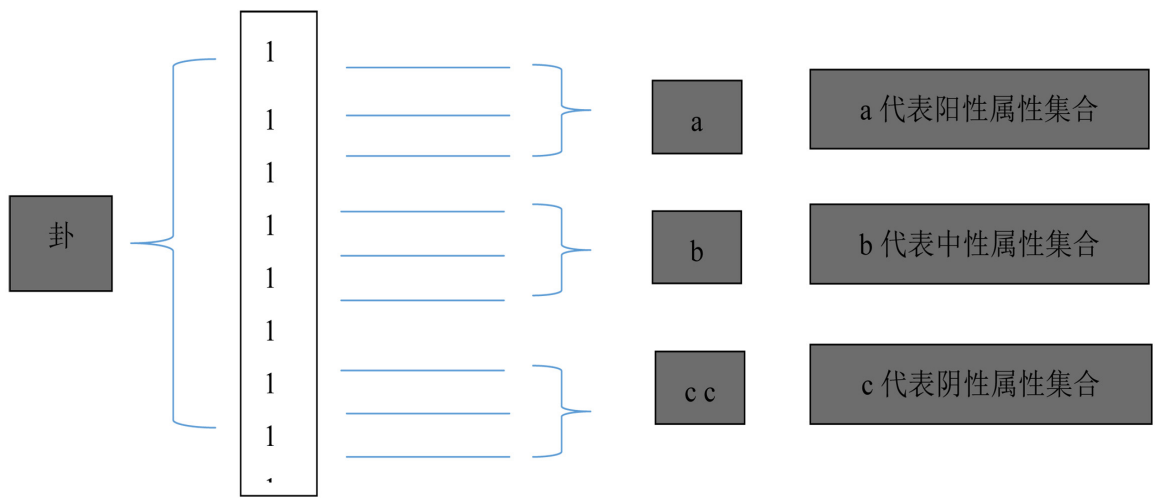

Figure 1. Gua

图 1. 卦
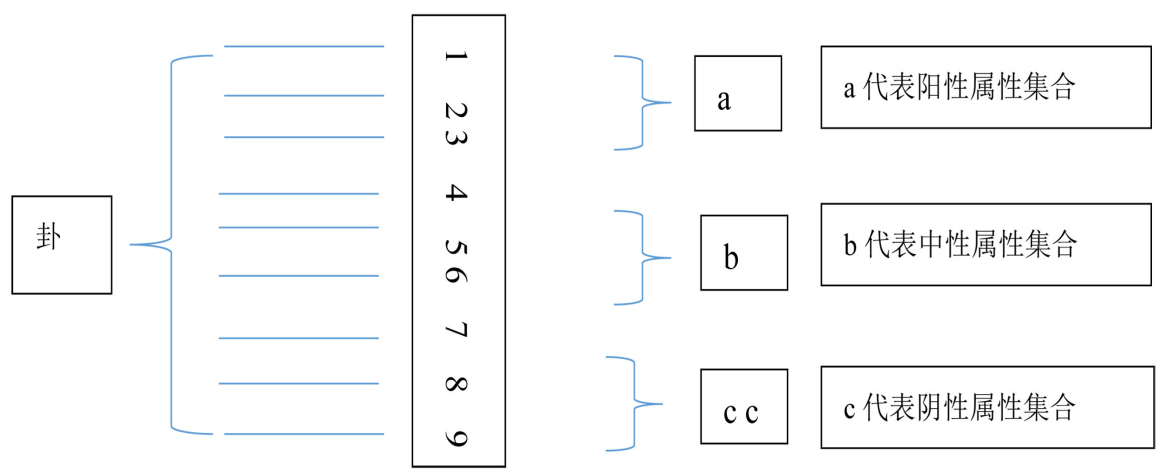

Figure 2. Gua 1

图 2. 卦 1 
素力量, 3,9 为阳爻表示正性或负性因素向中性因素转换的力量较强, 为阴 爻表示这种力量较弱。4 爻的阴阳代表中性因素向正性因素发展力量, 6 爻的 阴阳代表中性因素向负性因素发展力量。5 爻的阴阳代表中性因素的稳固程 度。

“-.-” 是阳爻代表影响因素强。“- - ” 是阴爻代表影响因素弱。阳爻有 两种长度 “--.-”、“---”, 阳爻长的代表影响因素对对象的运动发展影响 过强(对于卦一般我们只取较短的阳爻, 特殊情况下我们才用长的阳爻, 当长 阳爻出现时, 也就是所考察运动对象的运动平衡即将打破, 所考察对象的现 有状态即将崩溃)。

如: 1: 左侧文本框对应每个爻的数字代表的是这个爻的力量强度, 1 是 基本强度, 2 是中等强度 3 是超强(过强)。有了它我们能更好的表达各种影响 因素的作用变化关系。

\subsection{2. 主卦、变卦、及次卦}

每个考察对象的卦象包括主卦、变卦、及次卦, 最简单的卦象只包括主 卦。但要更全面、细致的思考问题, 则应该包括主卦、变卦、及次卦。

主卦是指被考察对象当时的卦象。

为了更好的考察一些影响因素, 我引入了次卦的概念。次卦是一些主要 影响因素与次要影响因素的卦象。次卦是对各种影响因素等等所作的卦。当 然我们只是选择性的作出。

为了考察对象的运动变化特点, 我引入了变卦的概念。变卦也可叫因果 卦。变卦包括主卦的因卦。主卦及对象在现有卦象下, 自主或调控后运动变 化形成的结果所对应的卦象就是变卦。也就是主卦的前因后果。

为了更好的解决问题, 我们需要考察影响对象运动变化的各种因素, 及 对象在这些因素影响下的运动变化规律, 及我们应该采取的策略。作出主卦、 次卦表示我们考察到了影响对象运动变化的各种因素, 及它们相互影响变化 发展规律, 而作出变卦表示我们考察了对象在这些因素影响下的运动变化规 律, 及我们应该采取的策略。

a) 卦名、卦辞，爻名、爻辞、卦注

每个卦仍然有卦名、卦辞, 爻名、爻辞, 卦辞分为总卦辞, 分卦辞, 具 体卦辞, 爻辞分为总爻辞, 分爻辞, 具体爻辞, 相同爻位如果爻相同, 则总 爻辞相差不大, 分爻辞是一类对象集合的爻辞(比如疾病相关对象集合, 与社 会发展相关对象集合, 我们就可能给出不同的爻辞, 当然还会有更细分对象 的爻辞), 具体爻辞是我们作每个具体卦时所列举出来各种影响因素所对应的 爻位及变化关系的相应说明。下层卦辞、爻辞一般是上层卦辞、爻辞的具体 应用。

每个卦还有卦注, 每个卦注都要标注一些注意事相, 以指导我正确或创 造性的思考问题。卦注也分总卦注, 分卦注, 具体卦注。下层卦注一般是上 层卦注的具体应用。

b) 如图 3: 如果被考察对象运动的正性方向是我们希望的方向。那么理 想状态下应该正性因素作用强 (与这句话对应的爻为 1 爻, 1 爻为强阳爻), 负 


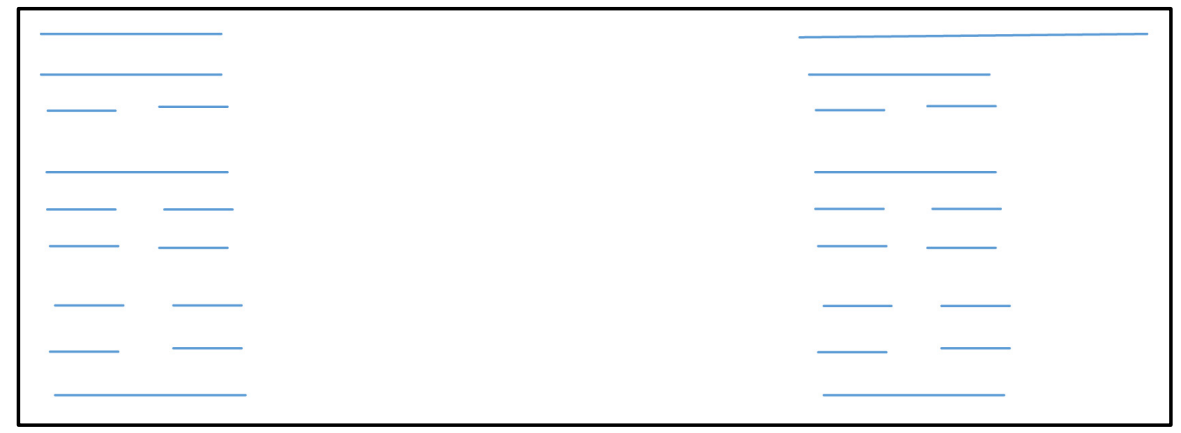

Figure 3. Example of relationship between the lines and the object movement 图 3. 举例爻与对象运动的关系

性因素作用弱 (7 爻为阴爻), 同时次要正性因素不断转化为主要正性因素(2 爻 为阳爻), 中性因素亦不断向正性因素转化 (3 为阴爻, 4 爻为阳爻, 5 爻为阴 爻)。其它因素向负性因素转化变弱(6 爻为阴爻), 并且负性因素不断向中性 或者正性因素转化, 主要的负性因素不断成为次要的负性因素 (8 爻为阴爻, 9 爻亦为阳爻), 也就是说正性力量不断变强, 负性力量不断变弱。这样对象才 会向正性方向运动。

对应上述描述的理想状态下的爻： 1 为强阳爻， 3 为阴爻。 2 爻为阳爻。 4 爻, 9 爻亦为阳爻, 次要因素转为主要因素及中性因素转为正性因素活跃。 一爻为强爻。7 爻为阴爻。8 爻为阴爻。5 爻为阴爻, 6 爻为阴爻。这些爻所 代表的运动状态只是我们对目标对象所希望达到的, 也是我们努力达到的目 标状态。

c) 举例说明

如图 1: 这个卦的卦名是乾卦。

1) 卦注(我是根据我的经验添加的卦注, 今后是可以修改的): 一、总卦 注(是所有卦都具有的): (1) 首先各种卦、爻都是我们根据我们对考察对象的 认知而作，对对象的认知的不同是会作出不同的卦爻的，这就使我们所作的 卦象与实际情况往往会有些出入, 但只要这些 “出入” 不是 “原则性” 的, 我们往往会采取正确的策略, 从而获得正确的结果。我们如果要获得考察对 象相对正确的卦象, 就需要采取普遍联系的观点、整体的观点, 并同时用发 展的观点, 找出所有一定程度上影响或可能影响对象运动变化的因素, 并考 察这些因素是如何影响的, 同时考察它们的影响强弱, 它们的转化或可能的 转化情况, 并将它们归入不同的爻中, 从而给出我们认知的卦象。(2) 对于一 些复杂性运动对象, 因认知及侧重点的不同, 不同的人作出的卦象可能千差 万别, 采取的策略也可能存在一些差别(主要影响因素应该相同), 但最终的 结果可能相同, 也就是说条条大路通罗马。当然, 如果我们作卦时遗漏了重 要的影响因素, 那么作出的卦就是错误的卦; 易经只会给你提示, 给你类比 经验, 促使你更好的思考, 从而采取更合理的行为, 但并不会给你评判。这 种差异性也是创新的源泉, 所以不会评判。应该牢记的是易经的各种辞, 只 是他人的经验与总结, 对我们有重要的借鉴作用, 但仍然应该以我们自己为 主, 要创新就不能拘泥于这些经验与总结。二、分卦注(具体到某个卦及这个 
卦所对应的某类事例所应注意的)。三、具体卦注(某个具体的事例所应注意 的)。

2) 其卦辞是: 一、总辞: 乾卦表示被考察对象处于活跃状态, (如都是 1 倍或 2 倍爻, 且 1 爻和 7 爻的倍数相同, 那么对象处于平衡状态。如都是 3 倍爻, 那么对象因过于活跃, 而处于随时崩溃状态。)。一般情况下, 我们应 该(1) 促使中性因素向正性因素, 而压制它们向负性因素发展。(2) 压制负性 次要因素的活性, 而促进正性次要因素向正性主要因素发展。(3) 提高主要正 性因素的活力, 而压制负性主要因素的活力。二、分辞: 描述的是 1 社会学 领域。2 医学领域。…… 三、具体辞 1.1 (社会学领域的励志奋斗卦)要实现 我们的理想, 首先要不断考察理想实现的规律及我们现在的状况, 然后不断 行动起来, 在各种困境中不断锤炼自己, 一方面不断提高自己实现理想的各 种能力, 另一方面不断改变环境, 不断增加实现理想的力量, 不断消弱影响 理想实现的各种力量, 最终发生质变, 理想实现。

3）爻:一、总爻辞。如图 2: 1 所对应的爻, 代表的是这个对象向一方 向发展的主要正性因素, 1 爻是阳爻, 表示正性发展的力量强。7 所代表的是 主要负性因素, 7 爻是阳爻, 表示负性发展的力量也强, 1 爻与 7 爻都是 1 倍 阳爻, 说明这个对象处于平衡状态中; 2 与 8 所代表的是次要因素(也就是相 对中性因素)。2 爻为阳爻, 表示次要因素向主要因素转换的倾向较强。8 爻 为阳爻, 表示次要负性因素向主要负性因素转换的倾向较强; 3,9 分别代表 促进所在卦向中性或者相反方向发展的因素力量, 3, 9 为阳爻表示这种力量 较强。4 爻为阳爻代表中性因素向正性因素发展力量较强。6 爻为阳爻代表中 性因素向负性因素发展力量较强。5 爻的阴阳代表中性因素的稳固程度。二、 分爻辞。(比如医学领域各个爻所代表的意义就是具体到医学领域的分爻)三、 具体爻辞。(比如具体病例各个爻所代表的意义, 就是具体爻辞)

关于次卦、变卦见下面的具体事例。

\section{3. 具体事例}

我们日常作卦只作卦象、具体卦辞、具体爻辞或变卦, 在作卦的过程中, 我们能通过与易经 “数据库” 对比来获得相应卦象的总、分、具体的注、卦 辞及爻辞、变卦的共享 “数据” , 然后通过这些数据(事例、解释、提示等), 来进一步指导我们的作卦与思考。

1）通过卦与爻来更好的预测考察对象的发展变化。2) 是通过卦爻来思考 如何来更好的调控考察对象的运动发展, 使其向我们希望的方向发展。

(1) 2019 冬季我收了一个因咳喘七天入院的老病号, 他有慢性阻塞性肺 病, 常年咳喘, 每年要在我手里多次住院, 身体较差, 每次给予抗菌、消炎、 平喘、吸氧治疗几天就好转出院。但这次患者在治疗的中途因腰椎病出现腿 痛, 行动比较吃力, 而且因天气变冷受凉, 随即患者出现咳喘加重, 我习惯 性认为是受凉后肺病加重, 加强了抗菌、消炎、平喘、吸氧, 但患者病情未 见好转, 反而咳喘加重, 最后出现典型心衰症状后, 按心衰治疗同时给予抗 菌、消炎、平喘、吸氧治疗, 患者病情迅速好转出院。事后总结, 患者应该 在出现腰椎病并受凉感冒后, 心衰就产生, 当时没有引起我的注意, 否则就 
不会出现典型心衰症状。

(2) 对于疾病, 治疗后, 如果所有的爻都是阴爻, 表示痊愈, 而如果所有 的爻都是阳爻，且它们的倍数在 1 到 2 之间，表示正处于高峰，但是稳定的， 如果倍数是 3 , 表示身体可能会不耐受。

由于这个病人所患慢性病无法根治, 且其致病因素更多情况下是无法耐 受一些正性调控(比如免疫因素), 我们一般采取权宜方法, 就是通过药物一 方面来压制一些 “过度” 反应(机体无法耐受), 另一方面调高正性因素, 最 后只要病情稳定下来, 不进一步发展就算达到治疗效果。

(3) 对于这个患者, 影响病情发展的因素设计到致病因素的强弱(这里指 病菌等等)、患者的身体状况、药物的使用、患者的心理及生活习惯、天气等 等。使病情向好的方向发展的因素(肝、肾的功能处于相对良好的状态, 天气 晴朗, 患者的依从性及积极的心态, 正确用药), 使病情向坏的方向发展的因 素(肺处于一个不好的状态、心脏亦受影响, 情绪低落, 天气突然变冷, 药物 使用不当等等)。主要影响因素是药物与身体状况。

如图 4 是习惯性认知的变卦。

因为是老病号, 就没有认真考察各种影响疾病发展的因素, 而习惯性沿 用以前的治疗方法。没有认真考察患者的各种影响病情发展的因素, 潜意识 里的卦象是(如图 4 中的第二卦图): 1 爻是 2 倍阳爻(机体的免疫因素及调节 呼吸因素大大增强, 抗菌消炎、平喘等药), 2 爻是阴爻(潜意识认为用药后就 会好转, 次要的影响因素如天气、生活习惯、心脏功能等被我忽略, 认为转 化为主要影响因素的可能性较小)。3 爻是阴爻。4 爻是阴爻(潜意识认为肝、 肾的功能都好、一些心理认识、一些饮食, 不会成为影响因素)。5 爻是阳爻(同 4 爻认识, 说明中性因素比较稳定)。6 爻是阴爻(同 4 爻认识)。7 爻是 3 倍阳 爻(在强致病因素的影响下, 患者身体处于接近崩溃状态, 病菌毒力较强同有 抗药性, 机体免疫机制反应过度)。8 爻是阴爻(潜意识认为用药后就会好转, 次要的影响因素如天气、生活习惯、心脏功能等被我忽略, 认为转化为主要 影响因素的可能性较小)。9 爻是阴爻。

潜意识认为通过抗菌消炎, 降低机体的过度反应, 对症支持治疗, 患者 的卦象会是 1 爻成为 3 倍阳爻和 7 爻成为 2 倍阳爻(机体反应强度下调, 药物 影响因素加强了), 其它爻不变, 治疗几天后 1 爻成为 2 倍阳爻, 而 7 爻成为 1 倍阳爻, 而 3 及 9 爻成为阳爻 (次要影响因素开始向中性影响因素转变), 而 当减药几天后仍然是这样的爻象就可以带药出院了。

在咳喘加重, 而我认为是受凉后肺病加重的时候, 我潜意识的卦象是 7 爻重新成为 3 倍阳爻, 8 爻也成为阳爻, 心脏影响因素仍然包含在 8 爻中。 但实际情况是心脏影响因素已经成为主要影响因素, 包含在 7 爻。由于没有 针对心衰进行专门用药, 结果心衰加重, 心脏影响因素越来越强, 最后几乎 威胁到生命。

(4) 但如果我用易经来思考, 就要仔细考察各种影响因素及相互影响变化 关系, 这样我就会更多的给予患者的心肝肾功能及其它影响因素的关注, 特 别是心脏, 2 爻、6 爻及 8 爻就会变为阳爻(潜意识认为那些次要因素影响不 大, 但考察后看法会发生一些改变, 会给予更多关注), 其卦象, 如图 5 的第 


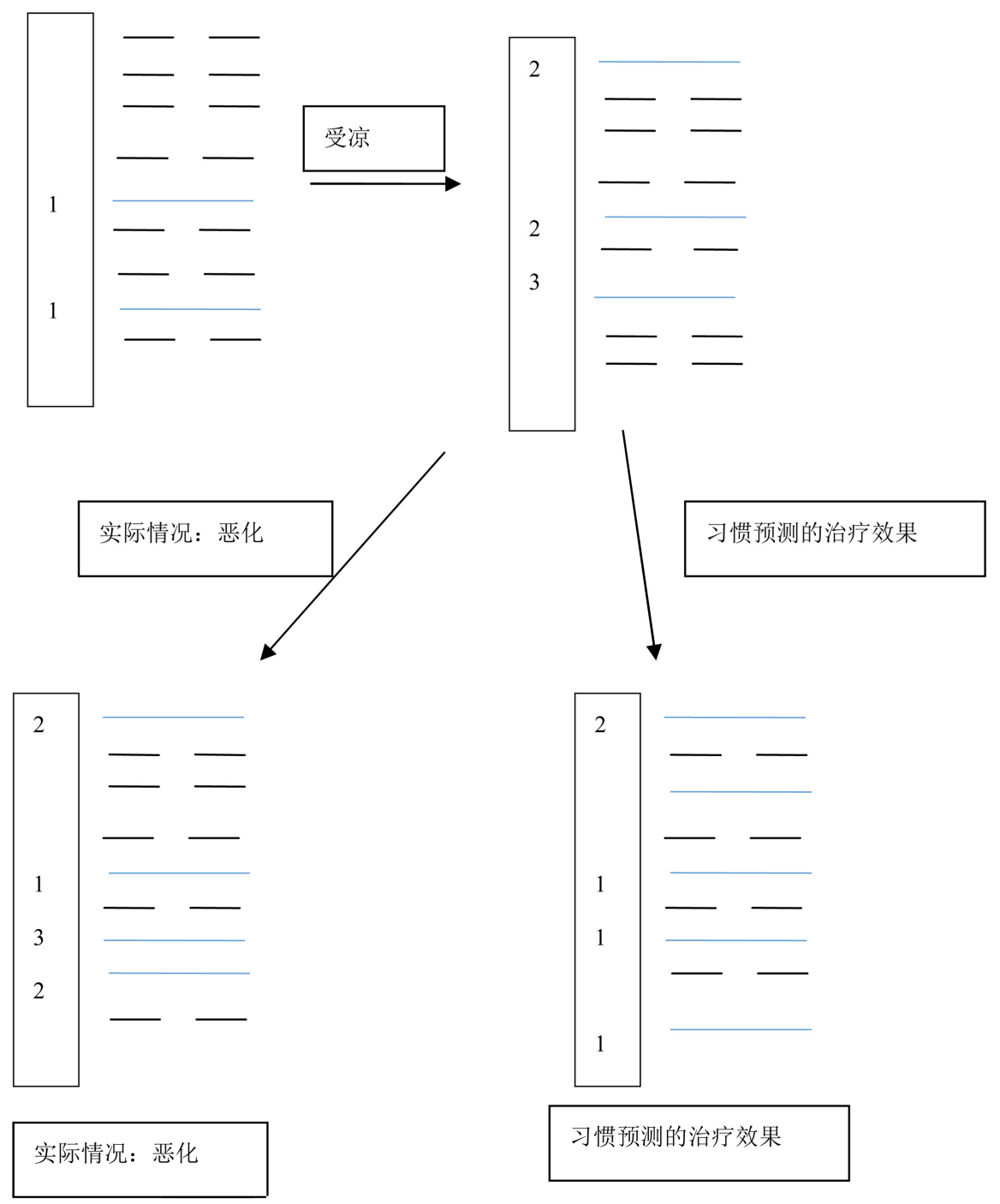

Figure 4. The change gua of disease 图 4. 病情变卦

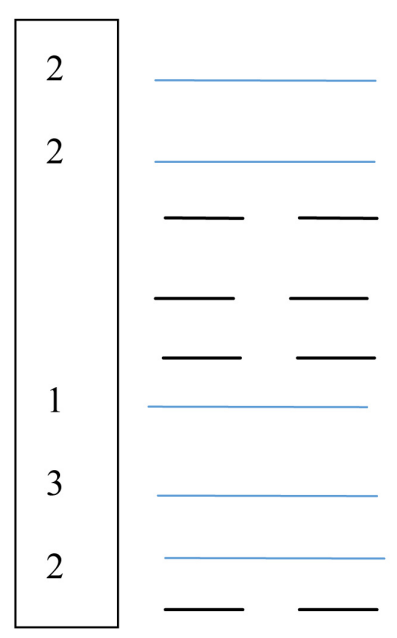

Figure 5. The primary gua of cardiac function 图 5. 心功能主卦 
二卦图, 它也是主卦象。当治疗过程中病情加重的时候, 我就会有更大的可 能提前发现已经心衰, 心脏成为主要致病因素, 其第 7 爻就不会最终发展成 为大阳爻, 而使其卦象处于崩溃状态时才发现。

在这种情况下, 我们医生一般会预防性的给予强心药, 同时尽量减少一 些诱发心脏病的因素。8 爻的倍数就会下降。抽象出来治疗策略是, 对于一 些无法根除的容易成为主要影响因素的次要影响因素, 我们可以采取压制它 成为主要影响因素的趋势, 也可以去除或压制诱发它成主要影响因素的因素。 抽象出来后我们就可以在其它领域进行运用, 比如社会领域如果有对象是相 似卦象……

图 6 是心脏的卦象, 是次卦, 它的 1 爻与 7 爻都是 1 倍爻处于平衡状态, 但是一些主要或次要因素可能随时进一步发生变化而使 7 爻向 3 倍爻变化, 比如咳喘进一步加重或天气进一步恶化等等都加重心脏负担, 从而使平衡打 破而崩溃, 发生心衰, 心脏因素就会从次要因素变为主要因素。

本事例卦注(我是根据我的经验添加的卦注, 今后是可以修改的): 一、 总卦注(是所有卦都具有的, 是易经具有的, 不是作具体卦象时所作的): 1) 首 先各种卦、爻都是我们根据我们对考察对象的认知而作, 对对象的认知的不 同是会作出不同的卦爻的, 这就使我们所作的卦象与实际情况往往会有些出 入, 但只要这些 “出入” 不是 “原则性” 的, 我们往往会采取正确的策略, 从而获得正确的结果。我们如果要获得考察对象相对正确的卦象, 就需要采 取普遍联系的观点、整体的观点, 并同时用发展的观点, 找出所有一定程度 上影响或可能影响对象运动变化的因素, 并考察这些因素是如何影响的, 同 时考察它们的影响强弱, 它们的转化或可能的转化情况, 并将它们归入不同 的爻中, 从而给出我们认知的卦象。2) 对于一些复杂性运动对象, 因认知及 侧重点的不同, 不同的人作出的卦象可能千差万别, 采取的策略也可能存在 一些差别(主要影响因素应该相同), 但最终的结果可能相同, 也就是说条条 大路通罗马。当然, 如果我们作卦时遗漏了重要的影响因素, 那么作出的卦

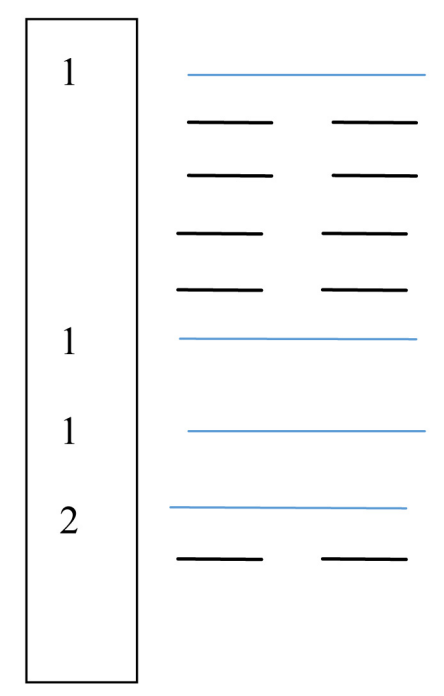

Figure 6. The secondary gua of cardiac function 图 6. 心功能次卦 
就是错误的卦; 易经只会给你提示, 给你类比经验, 促使你更好的思考, 从 而采取更合理的行为, 但并不会给你评判。这种差异性也是创新的源泉, 所 以不会评判。应该牢记的是易经的各种辞, 只是他人的经验与总结, 对我们 有重要的借鉴作用, 但仍然应该以我们自己为主, 要创新就不能拘泥于这些 经验与总结。二、分卦注(具体到某个卦及这个卦所对应的某类事例所应注意 的)。1…. $2 \ldots \ldots$. . 3、(表示前面还有一些卦注没有列举出来)一些次要致病 因素易发展为主要致病因素, 但因其引起的症状与另一主要致病因素引起的 症状相似而容易被忽略, 我们要特别注意这类次要致病因素。三、具体卦注(某 个具体的事例所应注意的)。1 ..... 2 2 ..... $3 \ldots . . .3 .3$ 对于慢性阻塞性肺病来 说, 易发生心衰……

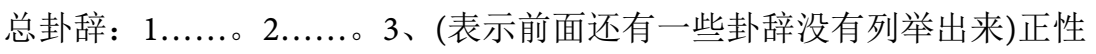
主要影响因素集合中的 A 因素, 它是系统自我产生的, 是随负性主要影响因 素的发展而发展的, 当 $\mathrm{A}$ 因素增强到一定程度后, 会超过系统的负荷, 而使 系统崩溃。我们采取的策略是: 压制 A 因素, 但必须同时调高 B 正性影响因 素(在系统负荷之内)。同时压制负性次要因素及中性因素, 不使它向负性主 要因素或负性因素发展。

分卦辞: $1 \ldots \ldots .2 \ldots \ldots .3 、 \ldots . .3 .3$ (医学有关) 人是一个平衡体, 当致病 因素致病时, 机体会自动产生一些因素(a)来去除这个致病因素的影响, 同时 这个(a)因素也会对机体产生影响, 如果超过了机体的承受能力, 便会对机体 的健康产生负性影响。我们采取的策略是, 对其它因素采取的策略不变, 对 因素 a 应该压制它的发展。

具体卦辞: $1 \ldots \ldots .2 \ldots . . .63 、 . . . .3 .3$ (医学有关) ......3.3.5.....3.3.5.3 (我 可以把这个具体病例按一定规律归入易经的卦辞中也就是数据库中)......(策 略方法前面已经论述)。

总爻辞: 1 爻为阳爻, 正性影响因素处于活跃状态。……2 爻……如图 8 列举了各种影响因素。左边图列举的是正性影响因素, 中间图列举的是中 性影响因素, 右图列举的是负性影响因素。

分爻辞: $1 \ldots \ldots$...... 2 . $3 . . .3 .3$ (医学有关) 1 爻为阳爻, 正性影响因 素处于活跃状态。 $\cdots \cdots \cdot 2$ 爻……

具体爻辞: $1 \ldots \ldots .2 \ldots \ldots .3 、 \ldots . .3 .3$ (医学有关) ......3.3.5.....3.3.5.3: 1 爻是阳爻, 通过使用抗菌消炎药控制感染……预防性的使用强心药……2 爻……

这个病例, 影响病情发展的因素设计到致病因素的强弱(这里指病菌等 等)、患者的身体状况、药物的使用、患者的心理及生活习惯、天气等等。使 病情向好的方向发展的因素(肝、肾的功能处于相对良好的状态, 天气晴朗, 患者的依从性及积极的心态, 正确用药), 使病情向坏的方向发展的因素(肺 处于一个不好的状态、心脏亦受影响, 情绪低落, 天气突然变冷, 药物使用 不当)。主要因素是药物与身体状况。其影响因素如图 9。

采取的策略, 调高正性动力、调低负性动力, 压制负性次要因素向负性 主要因素的转化。

1 爻主要正性因素, 肺部调控(zz1)、肺部用药(zzw1)。 
2 爻次要正性因素, 辅助用药(czw1)、天气好、心情(cz1)。

7 爻, 主要负性因素, 肺部因素(zf1)。

8 爻, 次要负性因素, 心脏(cf1)、肝肾、天气(cfw1)、心情等等。心脏因 素一定条件下随时可以转化为主要负性因素, 可以针对心脏预防性用药, 从 而降低其转化的可能 8 爻是 1 倍爻, 如果天气或其它加重心脏负担的因素发 生, 它可变为 3 倍爻, 随时可以转化为主要负性因素。

7 爻的 zf 表示主要内部负性动力。 zfw 表示主要外部负性动力

1 爻的 $\mathrm{zz}$ 表示主要内部正性动力, $\mathrm{zzw}$ 表示主要外部正性动力

8 爻的 $\mathrm{cf}$ 的次要内部负性动力, $\mathrm{cfw} \cdots \cdot . .$.

2 爻的 $\mathrm{czw}, \mathrm{cz}$ 表示的意义相似

如图 7 是这个病例用新易经分析后作的变卦。

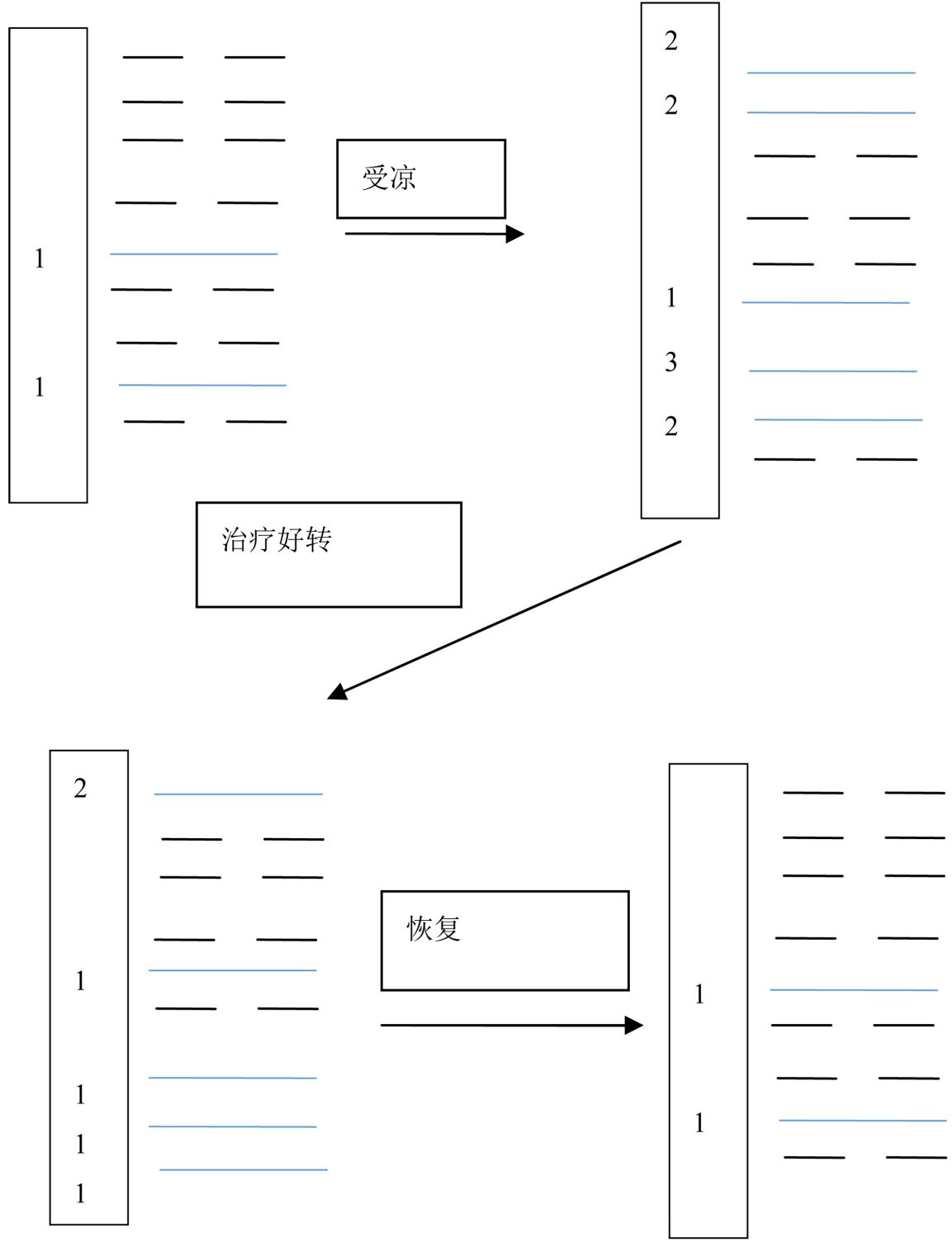

Figure 7. The change gua of case

图 7. 病例变卦 


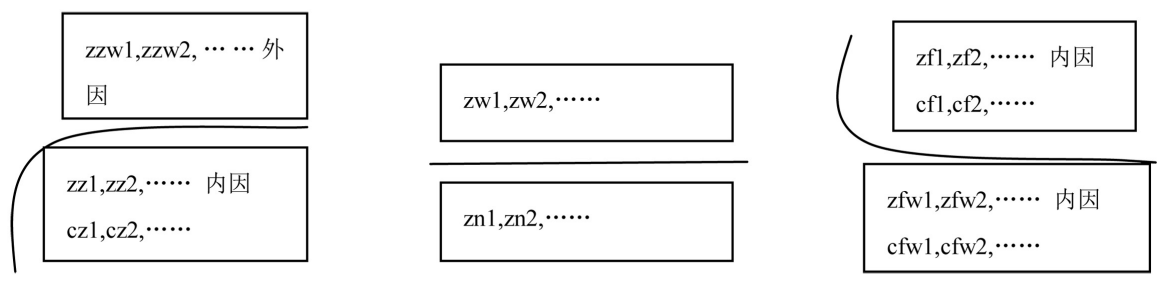

Figure 8. Data expression of the head remarks appended to the lines

图 8. 总爻辞的数据表示

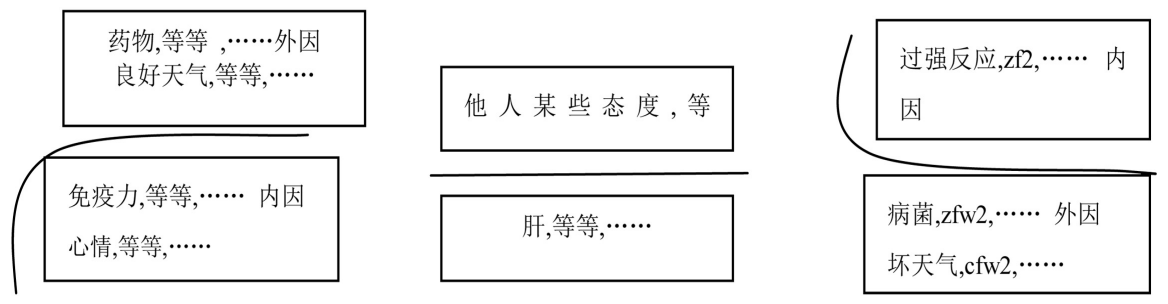

Figure 9. Data expression of the specific remarks appended to the lines

图 9. 具体爻辞的数据表示

\section{4. 运动数据库}

任何对象的运动都可以根据我们的目的, 选取一方向作为正性方向, 然 后选取一个标准状态, 在不同影响因素的作用下, 根据它的其它运动状态离 这个标准状态多远, 都可以按一定标准转化为与时间相关的距离, 并根据时 间与距离的关系获得其它属性, 如作用力, 从而作出它的运动轨迹。这样, 任何运动都可以转化为与时间相关的空间运动。我们在将运动对象的运动转 化为空间运动的过程中, 应该采取一定的标准, 同时卦辞、爻辞要尽量抽象 化、标准化, 这样就能将我们在一个具体的领域所获得的运动变化规律尽可 能方便且更多的应用到其它领域。图 8、图 9 是转化后的 “计算公式”, 每 个影响因素都代表一组计算, 链接到相应的运动数据库。当然这只是我的设 想。

我们大脑思考的任何运动对象都可以作出卦象。也就是说能被我们思考 的任何运动对象都可以用易经来进行归类。这样, 通过易经可以建成为一个 包罗万象的运动数据库, 任何运动对象我们都可以从易经的运动数据库中获 取对思考这个运动对象有益的信息。当建立了一个包罗万象的运动数据库后, 我们所要考察的任何一个对象都会在卦象库中找到相应的 “数据” (抽象的、 模糊的、具体的都会有), 从而使我们更快、更好的掌握所考察的对象。同时 根据卦象中的策略数据, 能让我们获得更多、更全面的策略。

\section{5. 小结}

当我们用新易经来思考某一问题时, 我们首先要明确思考的目的, 然后 根据目的选择思考的运动对象及运动对象的运动方向。有了对象与运动方向 后, 就能作出卦象。卦象除了反应考察对象的运动状态及发展趋势, 也反应 了各种影响因素相互影响及变化关系。因而, 为了作出相对正确的卦象, 在 作卦的过程中, 我们需要找出各种影响对象运动的因素, 并确定哪些是主要 
的, 哪些是次要的, 哪些是内因, 哪些是外因…… (以上的思考过程, 必然包 含普遍联系的观点、整体的观点、矛盾的观点), 需要弄明白这些因素的相互 影响发展变化关系(这种思考必然包含发展的观点), 还需要明白考察对象的 运动发展趋势。

作出卦象后, 我们就能通过对比获得新易经中相应的标准卦象, 并获得 相应的卦注、卦辞、爻注、爻辞、各种变卦以及各种经验和各种应对策略。 从而在我们思考遇到困难时给我们以启迪。使我们能更快、更好地掌握所考 察的对象。同时根据卦象中的策略数据, 能让我们获得更多、更全面的策略。 跨领域对各种运动数据的运用, 也有益于我们创造性地解决问题。

新易经同时应该是一个开放的运动数据库, 各种机构、组织都可以按照 一定的规则向其中添加运动数据。每个人也可以根据自己的经验按照一定的 规则向其所拥有的新易经中添加运动数据。新易经就应是一个包罗万象的按 照一定规则组织起来的既包含各种抽象的、模糊的、具体的 “经验” 数据, 也包含各种转换获得的空间运动数据的 “大杂烩” 运动数据库。

新易经包罗万象, 容易理解, 富有逻辑性, 有了它我们在作卦时会自觉 地用全面、整体的观点, 普遍联系的观点, 变化发展的观点, 来考察各种影 响因素及对象的发展变化规律及相互关系, 使我们能更好、更快、更有创造 性地解决问题。因而, 它是我们学习、思考、工作实践的有力 “科学” 工具。 它是一个新的科学研究领域, 本文只起到抛砖引玉的作用, 对卦辞、爻辞、 运动数据库……等等的更全面、更严谨、更标准化的描述, 需要今后更多的 研究。

有了新易经后, 当我们思维出现瓶颈时, 作作卦, 看看思维有什么短板, 或看看在相似卦象下其它领域的研究采取的什么策略……是不是很有用!

\section{Conflicts of Interest}

The author declares no conflicts of interest regarding the publication of this paper.

\section{References}

[1] 郭树森, 张吉良. 大道之源: 《周易》与中国文化 [M]. 长沙: 湖南师范大学出版 社, 1993. 


\section{Appendix (Abstract and Keywords in Chinese)}

\section{新易经与运动数据库}

摘要：易经要适应现代科技的发展就要对它进行修改。为了使它通俗易 懂, 成为我们学习、思考、工作实践的有力 “科学” 工具, 根据我们的思维 特点, 我尝试对易经的卦象、卦辞、爻辞重新进行了修改, 从而促使我们在 通过易经思考问题时必然会用全面、整体的观点, 普遍联系的观点, 变化发 展的观点, 来考察各种影响因素及对象的发展变化规律及相互关系, 使我们 能更好、更快、更有创造性地解决问题。

关键字: 新易经, 卦象, 主卦, 变卦, 运动数据库 